\title{
A COUNTEREXAMPLE TO THE EQUIVARIANT SIMPLE LOOP CONJECTURE
}

\author{
KARIN USADI
}

(Communicated by James E. West)

\begin{abstract}
In 1985 Gabai gave a complete proof of the Simple Loop Conjecture, which states that any map between closed surfaces, which does not induce an injection on the level of $\pi_{1}$, takes some noncontractible simple loop in the domain surface to a contractible loop in the target surface. In this paper we study an analogous result for the category of surfaces equipped with finite group actions and the maps which commute with the group structures (the "equivariant" maps). We find a counterexample to the equivariant analog of the Simple Loop Conjecture for the cyclic group of order 3 . The proof uses an equivariant analog of a theorem of Edmonds which gives a standard geometric representative for any homotopy class of surface maps of nonzero degree.
\end{abstract}

\section{INTRODUCTION}

The study of compact two-dimensional manifolds, or surfaces, led to a complete classification about 100 years ago. However, the maps between compact surfaces continue to be studied. For example, one can ask, does there exist a nice geometric representative for any homotopy class of surface maps?

In 1927 Nielsen [11] proved that a map between compact surfaces inducing an injection on the level of fundamental groups is homotopic to a covering map. Edmonds [1] extended this result in 1979, proving that any surface map of nonzero degree is homotopic to the composition of a degree one map, which collapses submanifolds with connected boundary to points, with a branched covering.

One corollary of this latter work was a partial solution to the then open Simple Loop Conjecture, which states that a map between closed surfaces, not homotopic to a covering map, takes some noncontractible embedded loop in the domain surface to a contractible loop in the target surface. In 1985 Gabai [5] gave a complete solution for closed surfaces.

It is known that the conjecture is false for surfaces with boundary. One can take, for example, the 2-fold branched covering of the annulus which may be described as follows. There is a 2 -fold branched covering from the unit disk

Received by the editors September 5, 1991.

1991 Mathematics Subject Classification. Primary 57M60; Secondary 57S25, 57N05.

Key words and phrases. Surfaces, two-dimensional manifolds, low-dimensional geometric topology, group actions on surfaces, equivariant maps between surfaces, simple loop conjecture. 
$D \subset \mathbf{C}$ to itself given by $z \stackrel{f}{\mapsto} z^{2}$. From this, one obtains a branched covering of the annulus by removing a small open disk $U$ from $\stackrel{\circ}{D}-\{0\}$, and its inverse image $f^{-1}(U)$.

One can study actions on surfaces by finite groups and attempt a classification of the class of maps which commute with the group structures (the "equivariant" maps). For orientation preserving actions, an equivariant analog of Nielsen's Theorem is true in general. (See [16] for a proof which follows from the theory of Fuchsian groups, [4, 7, 12] for a harmonic map approach, or [15] for a topological proof.) However, an equivariant analog of Edmonds' Theorem is true only for a certain class of groups (which includes the cyclic groups of prime power order) [15]. Furthermore, there exist counterexamples to the equivariant analog of the Simple Loop Conjecture even for the cyclic group of order 3, and one can use the equivariant analog of Edmonds' Theorem to prove this. This paper is an exposition of one such counterexample.

\section{Definitions and statement of a Counterexample}

Throughout this paper a surface $M$ will denote an orientable compact twodimensional manifold. A $G$-action on $M$ by a finite group $G$ is a monomorphism $G \stackrel{\rho}{\rightarrow} \mathrm{Homeo}^{+} M$, where $\mathrm{Homeo}^{+} M$ is the group of orientation preserving homeomorphisms of $M$. Given $m \in M$ and $g \in G$, we write $g \cdot m$ for $\rho(g)(m)$ and call $M$ a $G$-space. A map $f$ is equivariant or a $G$-map if for every $g \in G$ and $m \in M$ it satisfies $f(g \cdot m)=g \cdot f(m)$. A submanifold $X \subset M$ is invariant if $g \cdot X=X$ for every $g \in G$. A submanifold $X \subset M$ is equivariant if given any $g \in G$ either $g \cdot X=X$ or $g \cdot X \cap X=\varnothing$. A branched covering $\beta: M \rightarrow N$ is a finite-to-one map which is a covering except possibly over a finite number of points.

Let $G=\mathbf{Z}_{3}$, and $M$ and $N$ be closed surfaces of genera 6 and 3, respectively. Then $G$ acts on each of $M$ and $N$ with two fixed points, where the quotient surfaces of the actions are of genera 2 and 1, respectively (see Figure 1 in $\S 3)$.

Counterexample to the equivariant analog of the Simple Loop Conjecture. There is an equivariant 2-fold branched covering $\beta: M \rightarrow N$ not homotopic to a covering map, for which there is no equivariant noncontractible embedded loop in the kernel of $\pi_{1} \beta$.

\section{PreliminaRy discussion}

We need the following definitions.

Let $M$ be a $G$-space. The isotropy subgroup $G_{m}$ of an element $m \in M$ is the set of all elements of $G$ which fix $m$. The singular set of $M$ is defined by $S_{M} \stackrel{\text { def }}{=}\left\{m:\left|G_{m}\right| \neq 1\right\}$.

If $\beta: M \rightarrow N$ is a branched covering and $n \in N$ is a point over which $\beta$ is not evenly covered, then $n$ is called a branched point and $m \in \beta^{-1}(n)$ a singular point of $\beta$.

Remark 1. The Riemann-Hurwitz formula. The quotient map $M \stackrel{q_{M}}{\rightarrow}[M]$ of a group action is a branched covering. Thus, for a singular point $m \in S_{M} \cap(\partial M)^{c}$ there is a small disk $B_{m}$ which characterizes the $G$-action locally about $m$. 
More specifically, $B_{m}$ is invariant under the isotropy subgroup $G_{m}$, and is mapped off itself by every other element of $G$.

For example, in the smooth category, if $m \in S_{M}$ then the isotropy subgroup $G_{m}$ induces an orientation preserving action on the tangent space $T_{m} M$, which is given by $T_{m} h: T_{m} M \rightarrow T_{m} M$ for each $h \in G_{m}$. By averaging an arbitrary metric on $T_{m} M$, one may assume that $T_{m} h$ preserves length for every $h \in G_{m}$. Then $\left\{T_{m} h\right\} \cong G_{m}$ is a finite subgroup of $\mathrm{SO}(2)$ and therefore acts as a cyclic group of rotations in the plane.

Furthermore, the Riemann-Hurwitz formula [16]

$$
\chi M=|G|\left(\chi([M])-\sum_{i=1}^{k}\left(1-\frac{1}{l_{i}}\right)\right)
$$

is satisfied, where $\left\{x_{i}\right\}_{i=1}^{k}$ is the image in [M] of the singular set $S_{M}$, and $l_{i}$ is the order of the isotropy subgroup of the singular points mapping onto $x_{i}$.

Remark 2. Lifting homotopies. Note that an equivariant map $f: M \rightarrow N$ induces a map $[f]:[M] \rightarrow[N]$ between quotient surfaces. A homotopy $H$ is equivariant or a G-homotopy if $H_{t}$ is an equivariant map for every time $t$. If two maps $f$ and $f^{\prime}$ are equivariantly homotopic we write $f \sim_{G} f^{\prime}$.

An equivariant homotopy $H$ of $f$ induces a homotopy $[H]$ of $[f]$, but the reverse is not necessarily true. However, if for every time $t$ we have $\left[H_{t}\right]^{-1}\left(\left[S_{N}\right]\right)=[f]^{-1}\left(\left[S_{N}\right]\right)$ and $\left[H_{t}\right]=[f]$ when restricted to the inverse image of $\left[S_{N}\right]$, then by covering space theory $[H]$ lifts to a homotopy of $f$, and by the discreteness of $G$ the lift is equivariant.

Remark 3. Actions and representations. If $M$ is a $G$-space then there is an epimorphism $\rho_{M}: \pi_{1}\left(\left[M-S_{M}\right],[m]\right) \rightarrow G$ defined as follows. Let $m$ be a point covering $[m]$ under the quotient map $M \rightarrow[M]$. Given $\langle\alpha\rangle \in$ $\pi_{1}\left(\left[M-S_{M}\right],[m]\right)$, let $\alpha^{\prime}$ be the lift of $\alpha$ starting at $m$. Then $\alpha^{\prime}(1)=g \cdot m$ for some $g \in G$. We define $\rho_{M}(\langle\alpha\rangle) \stackrel{\text { def }}{=} g$. We call $\rho_{M}$ a representation of the action. Note that if $G$ is abelian then the element $g$ is independent of the base point $m$, and we may view $\rho_{M}$ as an epimorphism $H_{1}\left[M-S_{M}\right] \rightarrow G$.

Conversely, if $[X]$ is a compact surface, then any epimorphism $\rho: \pi_{1}([X],[x]) \rightarrow G$ determines a covering map $X \rightarrow[X]$ whose group of covering translations is $G$. (Take the cover corresponding to the subgroup $\operatorname{ker} \rho$ ). Furthermore, if $Y$ is the closed surface obtained by attaching a disk to each component of the boundary of $X$, the action of $G$ on $X$ extends over $Y$. If $C \subset \partial X$ is a component invariant under some $g \neq 1$, then the disk in $Y$ whose boundary is $C$ has a singular point fixed by $\langle g\rangle$. The Riemann-Hurwitz formula gives the Euler characteristic of $Y$.

Remark 4. Local cone branched coverings. A cone branched covering between disks viewed as unit disks in the complex plane is a map homotopic to $z \mapsto z^{r}$ for some nonzero integer $r$.

Let $M$ and $N$ be $G$-spaces, and $f: M \rightarrow N$ an equivariant map. For each $n \in S_{N}$, let $B_{N}$ be a small disk characterizing $n$ (see Remark 1 above). Then $f$ can be perturbed equivariantly and relative to the boundary so that it is a cone branched covering over each $B_{n}$. We call the result of such a homotopy a local cone branched covering over $S_{n}$ (see [15]). 
Furthermore, if $S_{M} \neq \varnothing$ and $[M]$ is a disk, then $f$ may be perturbed equivariantly so that $\left[f^{-1}\left(S_{N}\right)\right] \subset\left[S_{M}\right]$ (again, see [15]).

Remark 5. Local degrees. If $m \in S_{M}$ and $f$ is equivariantly homotopic to $h$, then by the discreteness of $G$ we have $f m=h m=n \in S_{N}$. Furthermore, if $f$ and $h$ are both local cone branched coverings over $S_{N}$, then the local degree of $f$ about $m$ is congruent modulo $\left|G_{n}\right|$ to that of $h$ about $m$. Also, for any $[w] \in\left[f^{-1}(n)\right] \cap\left[S_{M}^{c}\right]$, the local degree of $[f]$ about $[w]$ is a multiple of $\left|G_{n}\right|$ (see [15]).

Remark 6. An equivariant analog of Edmonds' Theorem. An equivariant pinch map is an equivariant degree one quotient map which collapses equivariant subsurfaces with connected boundary to points. Such a map is trivial if each equivariant subsurface it collapses is a 2-disk. Otherwise it is nontrivial.

Now, let $f: M \rightarrow N$ be an equivariant map between $G$-spaces of degree $d>0$. Then under suitable boundary conditions we have the following result.

Theorem [15]. Suppose that either $d=1$ or every element of $G$ has prime order. Then there is an equivariant pinch map $p$ and an equivariant $d$-fold branched covering $\beta$ such that $f \sim_{G} \beta \circ p$ relative to the boundary of $M$.

Note that cyclic groups of prime order satisfy the necessary hypotheses.

\section{Proof of the counterexample}

Let $M$ be a $G$-space. We define a simple loop $C \subset M$ to be a closed curve which is embedded with no self-intersections. An equivariant simple loop is a simple loop which is an equivariant submanifold. If $C$ is an equivariant simple loop, let $G C \stackrel{\text { def }}{=}\{g \cdot C: g \in G\}$.

Let $G=\mathrm{Z}_{3}$, and let $M$ and $N$ be closed surfaces of genera 6 and 3, respectively. Then $G$ acts on each of $M$ and $N$ with two fixed points, where the quotient surfaces of the actions are of genera 2 and 1, respectively.

Theorem (Counterexample to the equivariant analog of the Simple Loop Conjecture). There is a G-equivariant 2-fold branched covering $\beta: M \rightarrow N$, which does not induce an injection on $\pi_{1}$, for which there is no equivariant simple loop $C \subset M$ in the kernel of $\pi_{1} \beta$.

A brief outline of the proof follows.

By the lemma below, if there is an equivariant simple loop $C$ in the kernel of $\pi_{1} \beta$, then $\beta$ can be equivariantly perturbed so that $\beta(G C)$ is either one point or three points. Therefore $\beta$ induces a map $\beta^{\prime}: M^{\prime} \rightarrow N$, where $M^{\prime}$ is obtained from $M$ by cutting along each component of $G C$ and then collapsing the boundary components to points. By the equivariant analog of Edmonds' Theorem for cyclic groups of prime order (see Remark 6), $\beta^{\prime}$ is equivariantly homotopic to the composition of an equivariant pinch map $p$ with a branched covering $\alpha$. Since $\pi_{2} N=0$, the pinch $p$ must be trivial. Therefore $\beta^{\prime}$ is equivariantly homotopic to a branched covering $\alpha$. Then either $\left[M^{\prime}\right]$ is a torus, in which case $[\alpha]$ is a covering map, which is impossible because the local degree of $[\alpha]$ about each fixed point is congruent to 2 modulo 3 (see Remark 5), or $C$ cuts $M$ into two nontrivial pieces $M_{1}$ and $M_{2}$, and $\left.\alpha\right|_{M_{1}}$ is null-homotopic, which is impossible also for local degree reasons and by the inequality of Kneser. (See [17] for a statement of Kneser's inequality.) 
We proceed as follows.

Step 1. More detailed discussion of the $G$-actions on $M$ and $N$.

Step 2. Definition of $\beta$.

Step 3. There does not exist an equivariant simple loop $C$ in the kernel of $\pi_{1} \beta$.

Step 1 . Let $G=\{0,1,2\}$. $G$ acts on $M$ with two singular points $m_{1}$ and $m_{2}$, and on $N$ with two fixed points $n_{1}$ and $n_{2}$, where the representations for these actions are given by

$$
\rho_{M}\left[\partial B_{m_{2}}\right]=\rho_{N}\left[\partial B_{n_{1}}\right]=1 \text { and } \rho_{M}\left[\partial B_{m_{1}}\right]=\rho_{N}\left[\partial B_{n_{2}}\right]=2 \text {, }
$$

and $\rho_{M}\left(C_{i}\right)=\rho_{N}\left(K_{j}\right)=0$ for some set of homology generators $C_{i} \subset[M]$ and $K_{j} \subset[N]$, such that cutting along the $C_{i}$ 's and the $K_{j}$ 's cuts $[M]$ and $[N]$, respectively, into a single disk each (see Remarks 1 and 3 ).

Step 2. We define $[\beta]:[M] \rightarrow[N]$ to be a 2 -fold branched covering with two branched points $\left\{n_{1}, n_{2}\right\}$ and two singular points $\left\{m_{1}, m_{2}\right\}$, where the restriction $\left.[\beta]\right|_{\left[B_{m i}\right]}:\left[B_{m i}\right] \rightarrow\left[B_{n i}\right]$ sends $m_{i}$ to $n_{i}$ and is given by $z \mapsto z^{2}$ (where both $m_{i}$ and $n_{i}$ are identified with zero on the unit disk in the complex plane). The map $[\beta]$ lifts to an equivariant map $\beta: M \rightarrow N$. (See [15] for a detailed discussion of when a map between quotient surfaces lifts to an equivariant map.) Note that by the Riemann-Hurwitz formula, $\chi(M)=-2(6)+2=-10$ and $\chi(N)=-2(3)+2=-4$. In particular, $\beta$ cannot be homotopic to a covering map, since such a map would have to satisfy multiplicativity of the Euler characteristic. The map $\beta$ is shown in Figure 1.

Step 3. Suppose that there is an equivariant simple loop $C \subset M$ in the kernel of $\pi_{1} \beta$. By the lemma below, $\beta$ can be equivariantly perturbed so that $\beta(C)$ is a union of points in $N$. Since $C$ is equivariant, its image $[C] \subset$ $\left[M-\left\{m_{1}, m_{2}\right\}\right]$ is a simple closed curve.

There are two cases: either the components of $G C$ are freely permuted by the $G$-action, or $C$ is invariant under the $G$-action. We first fix some notation.

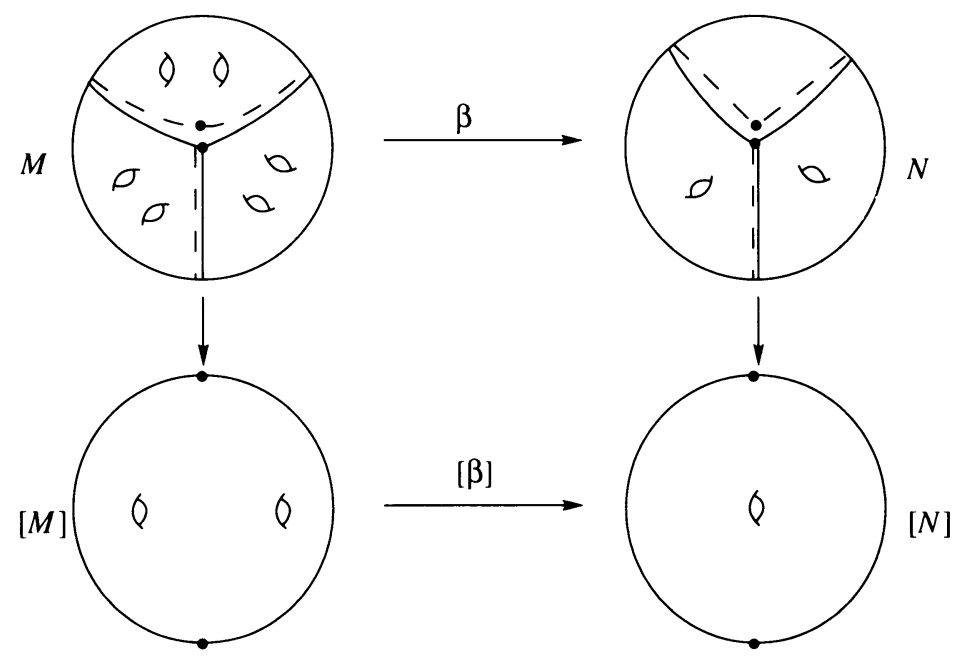

Figure 1 
If $G C$ is a union of noncontractible simple closed curves on a closed surface $M$, then by $M_{G C}$ we mean the (possibly disconnected) closed surface obtained from $M$ by first cutting along each curve in $G C$, and then taking the quotient space obtained by identifying each new boundary component of $M$ to a point.

Case 1. The components of $G C$ are freely permuted by the G-action. This means that $G C=\{C, 1 \cdot C, 2 \cdot C\}$ is a set of three disjoint curves. Then the map $\beta$ induces a map $\beta^{\prime}: M_{G C} \rightarrow N$ of degree 2 , where

$$
\chi\left(M_{G C}\right)=\chi(M)+6=-4>2(-4)=\left(\operatorname{deg} f^{\prime}\right)(\chi N),
$$

which contradicts Kneser's inequality. Therefore the following occurs.

Case 2. $C$ is invariant under the $G$-action. That is, $G C=C$. By the equivariance of $\beta$, since $C$ is a curve invariant under the $G$-action, $\beta C$ must be a fixed point. Therefore without loss of generality we may assume that $\beta C=n_{1} \in S_{N}$.

Case 2a. $C$ is nonseparating. Then there is an induced $G$-action on $M_{C}$, the closed surface of genus five, with exactly four fixed points $\left\{x, y, C_{1}, C_{2}\right\}$, where the $C_{i}$ are induced by the invariant loop $C$. The map $\beta$ induces a well-defined equivariant map $\beta^{\prime}: M_{C} \rightarrow N$, with $\beta^{\prime}\left(C_{1}\right)=\beta^{\prime}\left(C_{2}\right)=n_{1}$. We compute $\chi\left[M_{C}\right]$ using the Riemann-Hurwitz formula, $-8=\chi\left(M_{C}\right)=$ $3\left(\chi\left[M_{C}\right]-4 \frac{2}{3}\right)$, to conclude that $\chi\left[M_{C}\right]=0$. That is, $\left[M_{C}\right]$ is a torus. In particular, $[C]$ is a noncontractible curve in $[M]$.

By the equivariant analog of Edmonds' Theorem, $\beta^{\prime} \sim_{G} \alpha \circ p$, where $p$ is a $G$-pinch and $\alpha$ is a branched covering. Note that the map $[p]:[M] \rightarrow[N]$ induced by $[p]$ is a (nonequivariant) pinch map. Since $\pi_{2}[N]=0$, we conclude that $[p]$ must be homotopic to a homeomorphism. Therefore $[\alpha]$ is a 2 -fold branched covering between tori, and therefore by the Riemann-Hurwitz formula a covering map, so all its local degrees are equal to +1 . But the fixed points in $[M]$ mapped under $[\beta]$ into $[N]$ with local degrees are equal to 2 . These local degrees are fixed modulo 3 (see Remark 5), which leads to a contradiction. Thus the following occurs.

Case 2b. $C$ is separating. Then $C$ disconnects $M$ into two nontrivial pieces $M_{1}$ and $M_{2}$. Let $C_{1}$ and $C_{2}$ be the resulting two copies of $C$ so that $C_{i} \subset$ $M_{i}$. There is a $G$-action on the induced space $M_{1 C_{1}} \cup M_{2 C_{2}}$, where $\left\{C_{i}\right\}$ is a fixed point of its component. The $G$-action fixes each component $M_{i C_{i}}$. If $\left\{C_{i}\right\}$ were the only fixed point in $M_{i C_{i}}$, this would contradict the RiemannHurwitz formula applied to $M_{i C_{i}}$. Therefore by renaming we may assume that $m_{1} \in M_{1 C_{1}}$ and $m_{2} \in M_{2 C_{2}}$. Recall that $\beta(C)=n_{1}$. Therefore $M_{1 C_{1}}$ has two fixed points $m_{1}$ and $\left\{C_{1}\right\}$, each mapping onto $: l_{1}$, but has no fixed point mapping onto $n_{2}$.

Let $\beta_{i}: M_{i C_{i}} \rightarrow N$ be the maps induced by $\beta$. Since $M_{1 C_{1}}$ has no fixed point mapping onto $n_{1}$, by Remark 5 and Kneser's results about local degrees the degree of $\beta_{1}$ must be a multiple of 3 , and therefore must be 0 . Thus the degree of $\beta_{2}$ is 2 .

Since $C$ was noncontractible, it follows that $\chi\left(M_{1 C_{1}}\right) \leq 0$. Therefore by the Riemann-Hurwitz formula $\chi\left(M_{1 C_{1}}\right)=3\left(\chi\left[M_{1 C_{1}}\right]-2\left(\frac{2}{3}\right)\right) \leq 0$, which implies that $\chi\left(M_{1 C_{1}}\right) \leq 0$. Therefore $\chi\left(M_{1 C_{1}}\right) \leq-4$. That is, the genus $g\left(M_{1 C_{1}}\right)$ of $M_{1 C_{1}}$ is at least 3. But $g\left(M_{1 C_{1}}\right)+g\left(M_{2 C_{2}}\right)=g(M)=6$, so the genus $g\left(M_{2 C_{2}}\right)$ is at most 3. Therefore $\chi\left(M_{2} C_{2}\right) \geq-4$. Since the degree of $\beta_{2}: \chi\left(M_{2 C_{2}}\right) \rightarrow N$ 
is 2 , Kneser's inequality implies that $-4 \leq \chi\left(M_{2 C_{2}}\right) \leq 2 \chi(N)=2(-4)$, which is impossible.

It remains only to prove the following lemma.

Let $G$ be a cyclic group of prime order $p>2$. Let $M$ and $N$ be $G$-spaces with $N$ not a 2-sphere, and let $f: M \rightarrow N$ be an equivariant map.

Lemma. If $C \subset M$ is an equivariant simple loop in the kernel of $\pi_{1} f$, then there exists a tubular neighborhood $\nu$ of $G C$ in $M$ such that the restriction $\left.f\right|_{\nu}$ is equivariantly homotopic, relative to $\partial \nu$, to a map which is constant on C.

Proof. Since $G$ has prime order, either the components of $G C$ are freely permuted by the $G$-action or the loop $C$ is invariant under $G$. In the former case the desired result follows immediately by applying the Homotopy Extension Property (see [14]) to a small tubular neighborhood of $C$ and then extending equivariantly to each loop in the set $\{g \cdot C\}_{g \in G}$. So we suppose that $C$ is invariant under $G$.

Step 1 . The map $\left.f\right|_{C}$ extends equivariantly to a disk $D$ bound by $C$.

Proof. Since $\left.f\right|_{C}$ is null-homotopic, it can be extended to a disk $D$ whose boundary is $C$. We think of $D$ as the unit disk in the complex plane. Define $f^{\prime}$ to be $\left.f\right|_{D}$. The $G$-action on $C$ extends to $D$ as rotation by a $p$ th root of unity. This action has a unique fixed point $\{0\}$.

The goal is to change $f^{\prime}$ relative to $C$ into an equivariant map. If $f^{\prime} D$ does not contain a fixed point, then its image $\left[f^{\prime} D\right] \subset[N]$ is null-homotopic in $[N]-\left[S_{N}\right]$. This homotopy extends to a small tubular neighborhood $\nu$ of $[C]$ in $[M]$, relative to $\partial \nu$. This extended homotopy lifts equivariantly over $\nu$ by Remark 2. Thus the proof of the lemma is complete in this case. Therefore we assume that $f^{\prime} D$ does contain a fixed point, namely, $\left\{f^{\prime} 0\right\}$. Now $f^{\prime}$ is equivariant on $C \cup\{0\}$.

Let $g$ be any element of $G$. Note that $g^{-1} f^{\prime} g$ is a map from $D$ into $N$ which agrees with $f^{\prime}$ on $C \cup\{0\}$. Since $\pi_{2} N=0, f^{\prime}$ is homotopic to $g^{-1} f^{\prime} g$ relative to $C$. Since $(D \times I,(\{0\} \cup C) \times I)$ is a simplicial pair, the Homotopy Extension Property implies that $f^{\prime}$ is homotopic to $g^{-1} f^{\prime} g$ relative to $C \cup\{0\}$.

Since the action on $D$ is rotation, $D$ can be divided into $p$ equivariant sectors, each centered at 0 , which intersect pairwise along oriented rays $L_{\theta}=$ $\left\{t e^{i \theta}: t \in[0,1]\right\}$. Then for some element $g \in G$ the set of consecutive sectors is $\left\{S, g \cdot S, \ldots, g^{p-1} \cdot S\right\}$. Let $L$ and $g \cdot L$ be the rays bounding $S$. The boundary of $S$ is $L^{-1} *(g \cdot L) * \alpha$, where $\alpha$ is a connected oriented arc in $C$. This is illustrated in Figure 2.

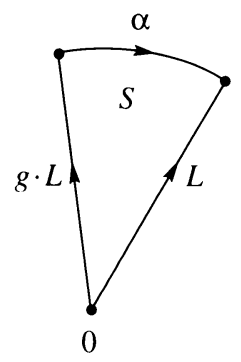

FIGURE 2 
Since $S \subset D$ is a subdisk, $\left.f^{\prime}\right|_{\partial S}$ is null-homotopic. Since $f^{\prime}$ is homotopic to $g^{-1} f^{\prime} g$ relative to $C \cup\{0\}$, we conclude that $\left.g f^{\prime}\right|_{L}$ is homotopic to $\left.f^{\prime} g\right|_{L}$ relative to the boundary of $L$. Define $f^{\prime \prime}: S \rightarrow N$ as follows. Let $\left.f^{\prime \prime}\right|_{\alpha \cup L}=$ $\left.f^{\prime}\right|_{\alpha \cup L}$ and $\left.f^{\prime \prime}\right|_{g \cdot L}=\left.g \cdot f^{\prime \prime}\right|_{L}$. Then $\left.f^{\prime \prime}\right|_{\partial S}$ is null-homotopic and therefore extends to a map on all of $S$. Now $f^{\prime \prime}$ extends equivariantly to $\left\{g^{k} \cdot S: k=\right.$ $2, \ldots, p-1\}$. That is, $f^{\prime \prime}: D \rightarrow N$ is an equivariant map which agrees with $f$ on $C=\partial D$.

This completes the proof of Step 1.

Step 2. The map $\left.f\right|_{D}$ is equivariantly null-homotopic.

Proof. Recall that $\left.f^{\prime} \stackrel{\text { def }}{=} f\right|_{D}$. By Remark 4 , since $p \neq 2$ implies that $C \cap S_{M}=$ $\varnothing$, the map $f^{\prime}$ can be perturbed equivariantly to be a local cone branched cover over a neighborhood of the singular set of the target surface. Furthermore, we can arrange so that $f^{\prime-1}\left(S_{N}\right)=S_{D}=\{0\}$. Now the perturbed $\left[f^{\prime}\right]$ is homotopic, relative to $\left[f^{\prime-1}\left(S_{N}\right)\right]$, to a map whose image is a small disk about $\left[f^{\prime}(0)\right]$. By Remark 2 this homotopy lifts equivariantly. Thus $f^{\prime}$ can be perturbed equivariantly so that $f^{\prime}(D)$ lies in a small disk about the fixed point $\left\{f^{\prime}(0)\right\}$. This entire disk can be equivariantly slid onto $\left\{f^{\prime}(0)\right\}$.

This completes the proof of Step 2.

Step 3. The equivariant homotopy of $\left.f\right|_{C}$ to a constant map can be extended to a homotopy over a tubular neighborhood $\nu$ of $C$ in $M$, relative to $\partial \nu$.

Proof. Let $\nu$ be a small tubular neighborhood of $C$ in $M$. By the Equivariant Collaring Theorem [3] we may arrange so that $\nu \cong S^{1} \times[0,1]$ has the product action, where $C$ is identified with $S^{1} \times \frac{1}{2}$. We obtain the desired homotopy $H$ as follows.

Let $\alpha$ be the connected arc on $C$ defined in Step 1. Recall that $C=$ $\bigcup_{i=0}^{p-1}\left(g^{i} \cdot \alpha\right)$, where $\left(g^{i} \cdot \alpha\right) \cap\left(g^{i+1} \cdot \alpha\right) \subset \partial\left(g^{i} \cdot \alpha\right)$. We now identify $\alpha$ with $\alpha \times \frac{1}{2} \subset S^{1} \times \frac{1}{2} \subset \nu$. Note that if $a \in \partial \alpha$ then $\partial \alpha=\{a, g \cdot a\}$. We define $H$ over $\alpha \times[0,1]$ and then extend equivariantly over the rest of $S^{1} \times I$.

Let $\left.H\right|_{\alpha \times 1 / 2 \times[0,1]}$ be the restriction to $\alpha$ of the homotopy of $\left.f^{\prime}\right|_{C}$ given by Step 2. Let $H(x, t)=f^{\prime}(x)$ for all $x \in \alpha \times\{0,1\}$ and all $t \in[0,1]$. Let $\left.H\right|_{\alpha \times\{a\} \times[0,1]}$ be any homotopy of $\left.f\right|_{a \times[0,1]}$ that agrees with $H$ over $a \times$ $\left\{0, \frac{1}{2}, 1\right\}$. Now extend $H$ equivariantly over $g \cdot(a \times[0,1])$. See Figure 3 .

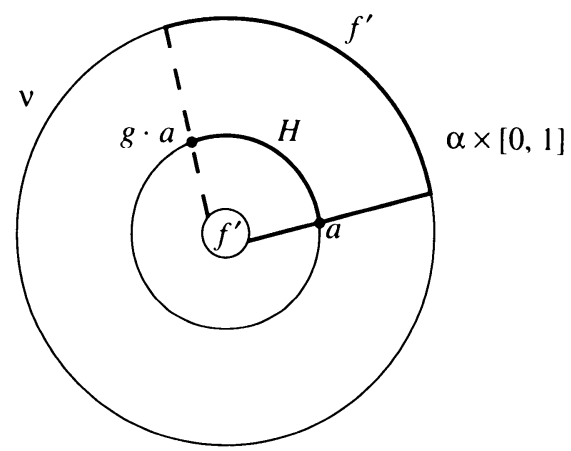

Figure 3 
Since $\left(\alpha \times[0,1],(\partial \alpha \times[0,1]) \cup\left(\alpha \times\left\{0, \frac{1}{2}, 1\right\}\right)\right)$ is a simplicial pair, the Homotopy Extension Property implies that $H$ extends over $\alpha \times[0,1]$. Finally, extend $H$ equivariantly over $\left\{g^{k} \cdot(\alpha \times[0,1]): k=2, \ldots, p-1\right\}$. This completes the proof of the lemma.

\section{ACKNOWLEDGMENT}

The results in this paper comprise part of the author's $\mathrm{Ph} . \mathrm{D}$. dissertation, written under the direction of Allan Edmonds at Indiana University [15]. The author is indebted to Professor Edmonds for his guidance in the preparation of these results.

\section{REFERENCES}

1. A. Edmonds, Deformation of maps to branched coverings in dimension two, Ann. of Math. (2) 110 (1979), 113-125.

2. __ Surface symmetry. I, Michigan Math. J. 29 (1982), 171-183.

3. G. Bredon, Introduction to transformation groups, Academic Press, New York, 1972.

4. J. Eells and J. H. Sampson, Harmonic mappings of Riemannian manifolds, Amer. J. Math. 86 (1964), 109-160.

5. D. Gabai, The simple loop conjecture, J. Differential Geom. 21 (1985), 143-149.

6. D. Gabai and W. Kazez, The classification of maps of surfaces, Bull. Amer. Math. Soc. (N.S.) 14 (1986), 283-286.

7. P. Hartman, On homotopic harmonic maps, Canad. J. Math. 19 (1967), 673-687.

8. J. Hempel, 3-manifolds, Ann. of Math. Stud., no. 86, Princeton Univ. Press, Princeton, NJ, 1976.

9. H. Kneser, Glättung von Flächenabbildungen, Math. Ann. 100 (1928), 609-617.

10. __, Die kleinste Bedeckungszahl innerhalb einer Klasse von Flächenabbildungen, Math. Ann. 103 (1930), 347-358.

11. J. Nielsen, Untersuchungen zur Topologie der geschlossenen zweiseitigen Flächen, Acta Math. 50 (1927), 189-358.

12. R. Schoen and S. T. Yau, On univalent harmonic maps between surfaces, Invent. Math. 44 (1978), 265-278.

13. R. Skora, The degree of a map between surfaces, Math. Ann. 276 (1987), 415-423.

14. E. Spanier, Algebraic topology, Springer-Verlag, Berlin and New York, 1966.

15. K. Usadi, On the classification of equivariant surface maps, Ph.D. dissertation, Indiana University at Bloomington, August, 1991.

16. H. Zieschang, Finite groups of mapping classes of surfaces, Lecture Notes in Math., vol. 875, Springer-Verlag, Berlin and New York, 1981.

17. H. Zieschang, E. Vogt, and H. Coldewey, Surfaces and planar discontinuous groups, Lecture Notes in Math., vol. 835, Springer-Verlag, Berlin and New York, 1980.

Ecole Polytechnique, Centre de Mathématique, F-91128 Palaiseau Cedex, France Current address: 17 rue Perdonnet, 75010 Paris, France

E-mail address: katz@iecn.u-nancy.fr 